\title{
"SMART PLANNING" - AN INTEGRATED APPROACH FOR DISTRIBUTION SYSTEM PLANNING TO COPE WITH ITS FUTURE REQUIREMENTS*
}

Christoph, ENGELS

FH Dortmund - Germany Lars, JENDERNALIK

WWE Verteilnetz - Germany Marc, OSTHUES

TU Dortmund - Germany
Heiko, SPITZER entellgenio - Germany christoph.engels@fh-dortmund.de lars.jendernalik@rwe.com marc.osthues@tu-dortmund.de heiko.spitzer@entellgenio.com

\begin{abstract}
Planning of Distribution Systems is facing several new challenges:

(1)The portion of decentralized renewable sources within the distribution area is increasing (leading to inversion of power flows and potential congestions).

(2)Prediction scenarios describing the future construction of renewable energy sources resp. decommissioning of conventional generation units are divergent and heterogeneous.
\end{abstract}

(3)The asset base mainly built during economic expansion periods within the last century are shifting to critical states.

(4) Investment budgets are shrinking.

An isolated treatment of each aspect cannot lead to a feasible approach since the objectives are in conflict to each other. This paper outlines an integrated computational approach for optimizing all aspects simultaneously. The solution is guided by actual planning principles of planning experts from the DSO partner.

\section{INTRODUCTION}

The traditional DSOplanning process tries to identify a target network structure for a given target year in the future [1]. In a deterministic world this approach leads to reasonable solutions. Unfortunately the uncertainty imposed by technical and political developments must be characterized as high in the actual situation [2]. To cover all possible future developments the network planer would have to construct a large variety of target network structures for each possible future situation. These structures may expose inconsistencies between the different targets. Even if the planer could manage these challenges the question which network structure should be really implemented is left open.

\section{APPROACH}

In order to address these challenges we propose

- to find the synergies between network expansion planning and strategic asset management (Figure 2).

- to make use of automation by computer models and optimization techniques.

- to combine the aspects of probabilistic scenario identification, network expansion planning, maintenance planning, contingency analysis, asset simulation, financial valuation and network optimization.

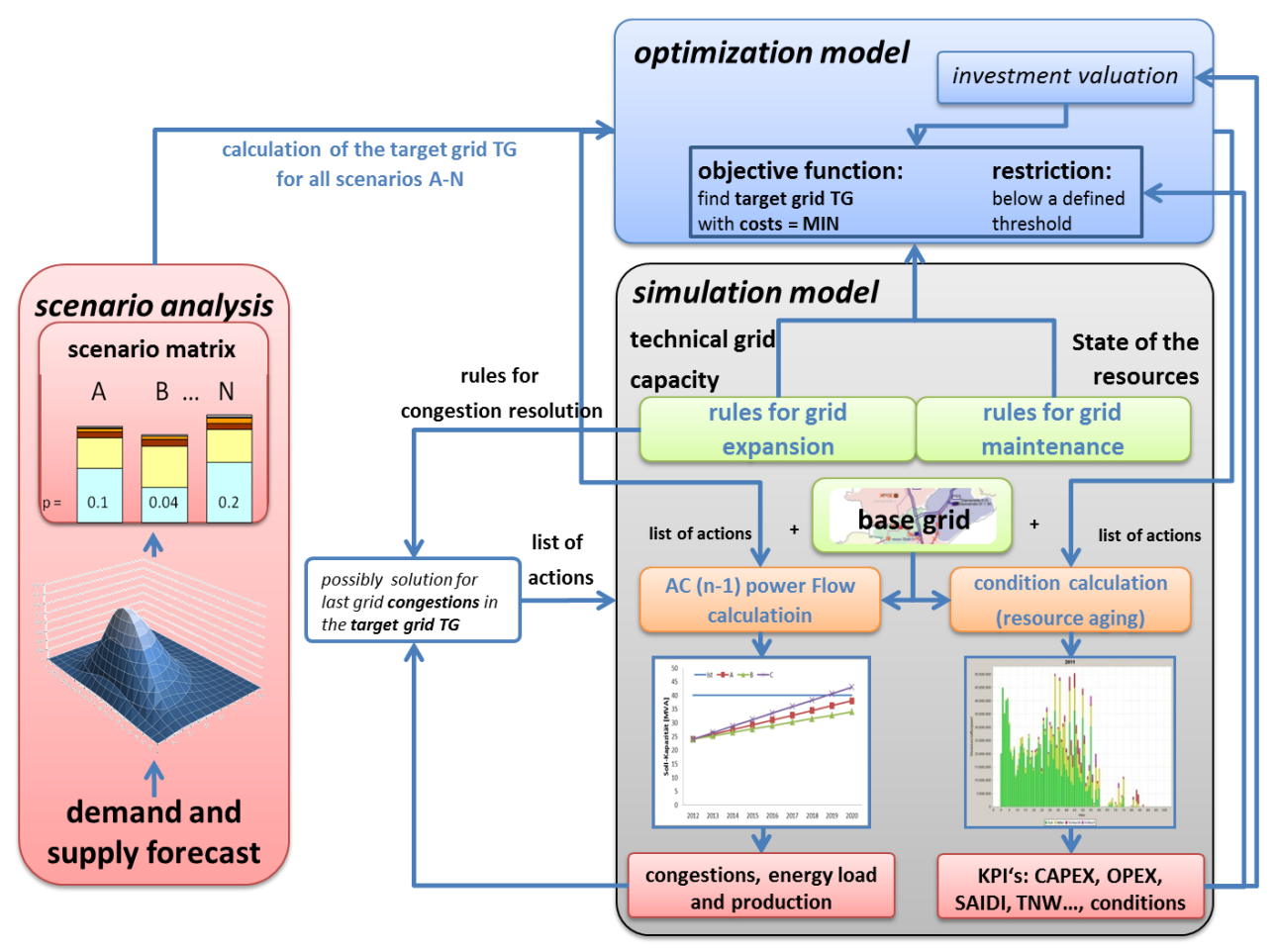

Figure 1: Architecture "Smart Planning” approach 


\begin{tabular}{|l||l|}
\hline $\begin{array}{l}\text { Asset-Management } \\
\text { (state based) }\end{array}$ & $\begin{array}{l}\text { Network-Development } \\
\text { (capacity based) }\end{array}$ \\
$\begin{array}{l}\text { Expansion of Asset-Simulation by } \\
\text { topological information }\end{array}$ & $\begin{array}{l}\text { inclusion of an uncertain future demand } \\
\text { and generation development (scenarios / } \\
\text { prediction models). } \\
\text { application of a power flow calculation. }\end{array}$ \\
\hline
\end{tabular}

Figure 2: Mergence of Asset Management and Network Planning

Figure 1 shows the proposed architecture starting with a prediction of demand and supply. We use public studies estimating the development of the energy mix on a coarse regional level. These studies provide the expectation value of the future development in demand (customers) and supply (renewable energy sources, conventional generation units as well as new technologies) which can be seen as the maximum in the distribution in Figure 1. From these estimations we derive a scenario matrix which reflects the uncertainty by specifying the variance in each energy source for each year throughout the prediction period.

\section{DEMAND AND GENERATION FORECAST}

The most likely scenario is provided by a DSO-driven demand and supply prognosis based on forecasts of regional demand and power generation potentials.

In presence of high uncertainty the most appropriate method of describing future developments is given by the scenario technique. Using the method one has to be aware of the fact that scenario technique is a weak prediction method also since the probability of each scenario has to be estimated by experts a priori. Nevertheless both the uncertainty imposed by changing regulatory as well as political constraints and variation in the energy mix disable alternative prediction methods.

Figure 3 shows the discretization of the expansion factor for demand and all regarded renewable energy sources in a dedicated year. The discretization is done to derive a set of possible states for a scenario generation. As depicted, the factors are given for a time interval of five years. A linearization between each value period provides an estimation of an annual development of the forecast. Based on this information, the time series for these expansion factors for all supplied regions is constructed in order to define a dedicated scenario.

Additional work in this project is the scenario generation. Based on the expected values given by the utilized study, probabilistic assumptions are made to generate a set of input variables (Demand, Wind, PV and Biomass) for each analyzed time step along the planning horizon and each scenario.

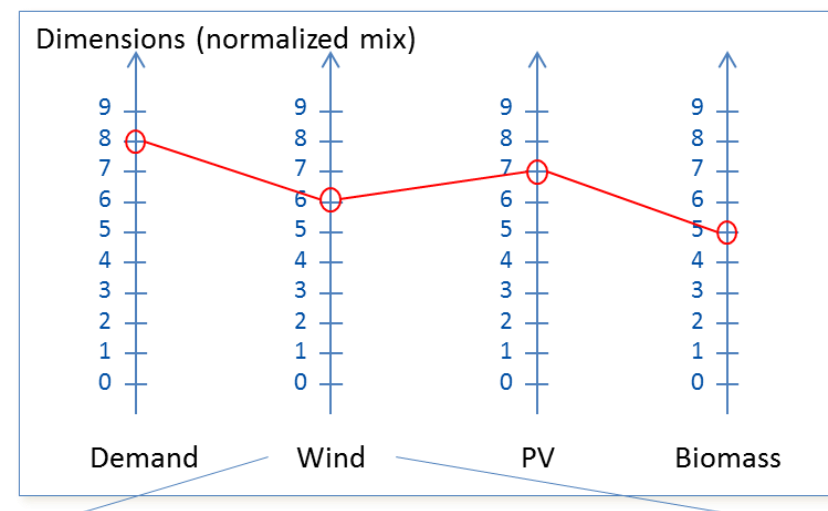

Predicted development per dimension per supply region

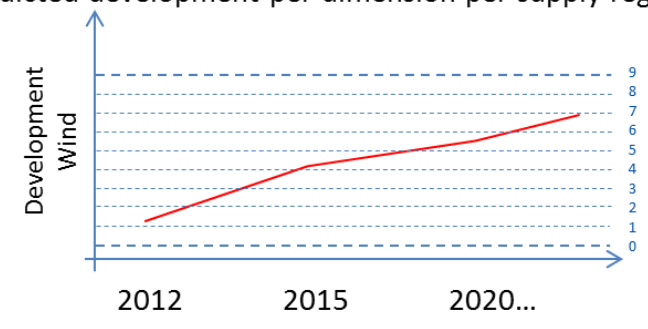

Figure 3: Scenario measures

\section{PLANNING RULE SET}

The integrated optimization approach tries to include expert knowledge in form of existing planning principles. These principles are modelled as formal planning rules which can be used by the optimizer to direct its search for optimal solutions and therefore decrease complexity. Figure 4 shows two examples for such rules: The precondition of the rule describes the actual situation which must be valid to activate the rule while the postcondition gives the situation after the rule has been applied. In the given example rules are shown which reinforce the capacity of a transformer station from 40 MVA to 60 MVA. While rule 1 uses an additional transformer of 20 MVA (which lead to a (n-1) secure capacity of $40 \mathrm{MVA}$ ), the second rule replaces the oldest transformer and adds a new transformer of 40 MVA (which results in a (n-1) capacity of 20 MVA only). Furthermore, the old transformer is added to the transformer pool. The transformer pool is a storage of transformers that life time has not been expired yet and provides flexibility for future congestions. Beside the treatment of transformers the rule set defines the extension of transformer stations also. In the given example the transformer station capacity remains constant providing space for 3 transformers in total per site. 


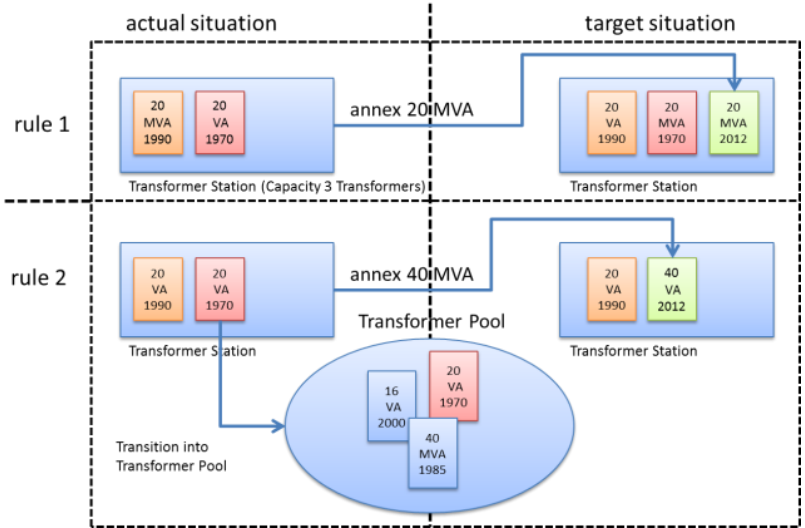

Figure 4: Excerpt from planning rule set

\section{POWER FLOW SIMULATION}

The compliance of the capacity constraints of the network elements are verified by power flow calculations (s.

). To solve the proposed optimization problem, the computation time has to be reduced for both, multiscenario calculations and for security analysis.

In providing (n-1)-security, the failure of each network element has to be taken into account. As computational effort is significantly increasing with the number of $\mathbb{N}$ network elements ( $2^{M}$ combinations), full AC load flow calculations are performed for the base case (n-0) and critical n-1-combinations. The latter are derived by the utilization of the DC load flow equations. A methodology is applied using Line Outage Distribution Factors (LODF) to estimate the influence of a line outage on the remaining elements [4]. The LODFs are calculated analysing the simplified admittance matrix by the use of the DC equations. Ex-ante, one DC power flow is performed determining the flow of the base case. Afterwards, the composition of the base case and the application of the LODFs results in a load flow for each failure. The critical outages are derived by filtering the line loadings using a defined threshold value (e.g. 90\%). First applications have shown a good approximation with an error about 2$5 \%$ depending on the size of the network structure.

As a result, the speed of n-1-contingency analysis is improved performing only a set of AC load flow calculations. Finally, the overall effect is given by the number of analysed set of scenario values. Hence, the simulation results provide the required information about location and size of capacity need (functional requirement).

\section{ASSET SIMULATION}

The foundation of the Integrated Optimization is modelled by time dependent relations within the dynamic Asset Simulation. The Asset Simulation provides an industry proofed tool $[5,6,7,8]$ which is based on the transparent method of cause and effect dependencies $[9$,
10]. The approach includes the definition of asset groups, objective measures and rules sets for feasible actions. The Asset Simulation applies an ageing process over all assets within the asset base assigning each asset to a state like (e.g. good, medium, critical). Depending on these states certain actions (like removal, new construction or replacement) are triggered. The IO.Netz approach needs a differentiation of the individual location of assets, for instance to include the effects of new generation sites. The realization of a location based representation of assets (left columns) and the ageing chain (upper right area) is shown in Figure 5.

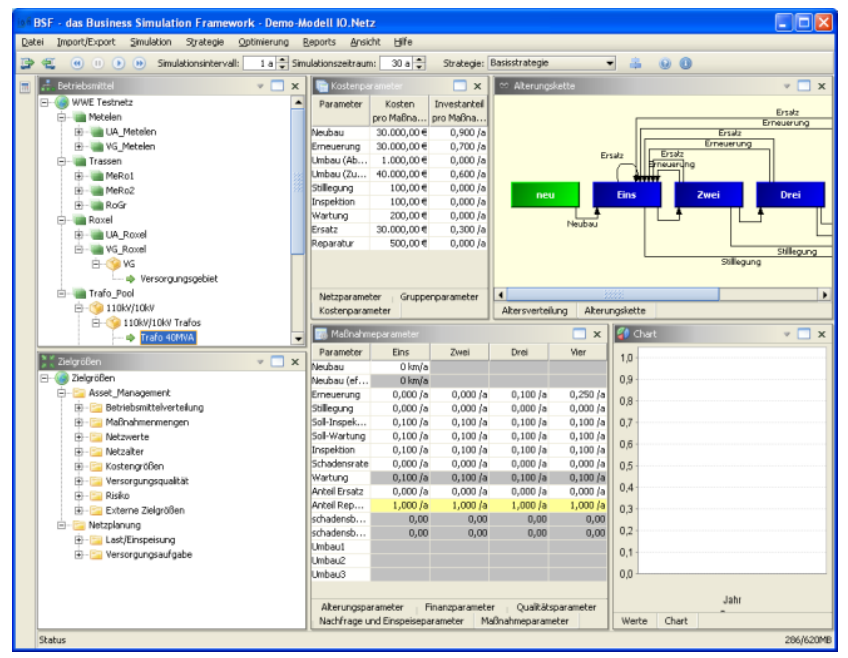

Figure 5: Asset Simulation GUI

\section{ASSET OPTIMIZATION}

The search space of an integrated optimization defined by a combination of the dimensions time, geography, asset type (defined in the given rule set) and multiple scenarios leads to a high complexity. Decision variables are given by discrete domain in contrast to [8]. The problem is further characterized by several restrictions:

- no contingencies

- limitations in global resources

- obligation to connect new suppliers

The principle of the asset optimization flow can be described by the following steps: The Dynamic Asset Simulation provides an objective measure (network budget, -quality, - risk, rev cap value) per scenario. The total objective measure is derived by a scenario weighted sum of target measures over all scenarios. Each scenario is further checked for contingencies and if a contingency is detected it is resolved by a scenario specific action from the rule set leading to additional costs.

The approach uses the stochastic optimization method of a Genetic Algorithm as a variation of Evolutionary Algorithms [12]. The application of user defined rule sets 
for actions within the network will narrow the final search space and thus leading to a reduced complexity.

\section{CASE STUDY}

The chosen approach used is derived from a planning process of a large regional DSO.

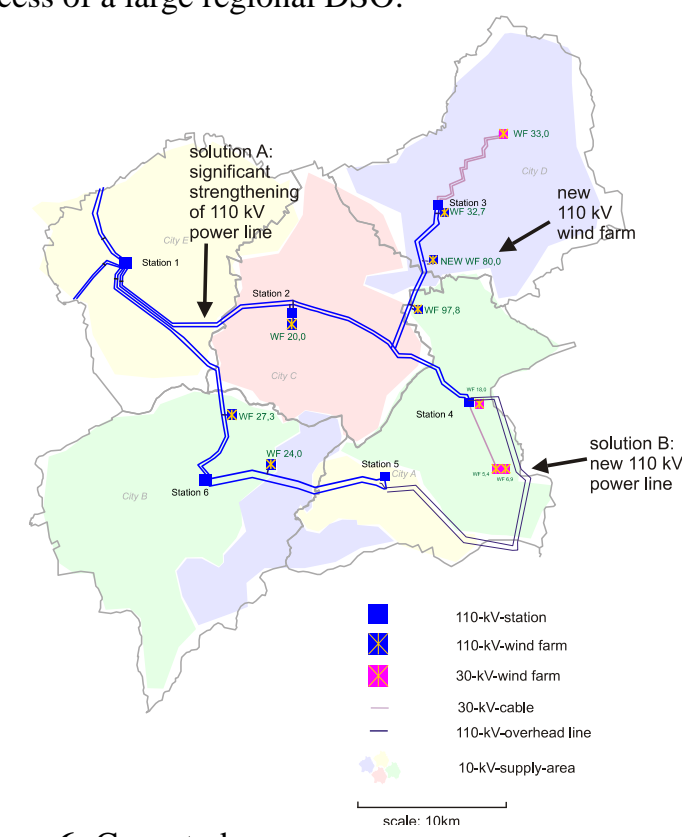

Figure 6: Case study

A first case study describes to following situation: An investor intends to build a new wind farm (or a repowering of an existing site) within the next 5-7 years (see Figure 6). To connect the wind plant to the power grid the construction of an additional power line will be necessary (e.g. a new $110 \mathrm{kV}$ line). The probability that the wind farm will be realized is given by $50 \%$. The existing power line within that region is outdated and cannot provide the capacity needed. The Asset Manager has to decide if the existing line should be replaced or a new line should be built with larger capacity.

The proposed system is designed to make the optimal decision between these two alternatives with respect to the given objective function.

\section{RESULTS}

The presented architecture is currently implemented by the research project IO.Netz $(*)$ which was introduced in the CIRED workshop 2012 [3]. In the meantime, first steps of this project were developed in a prototype including the interaction between asset simulation and optimization. This paper presents first results of the "Smart Planning" approach: contingency analysis, scenario matrix generation, and practical rule-sets for network expansion and maintenance planning.

\section{OUTLOOK}

The developed "new" integrated approach will be used for an analysis of a real distribution system. The practicability will be demonstrated by end of 2013 by integrating the different modules in the asset optimization process.

\section{REFERENCES}

[1] L., Jendernalik, Ch., Mensmann, M., Wohlfarth; "Target planning of electrical distribution grids as a fundamental module for a successful asset management", CIRED Workshop 2009, Prague, 2009.

[2] O. Krause, J. Schwippe, C. Rehtanz: Probabilistic Load Flow for Uncertainty Based Grid Operation, Proceedings of the 4th International Conference on Liberalization and Modernization of Power Systems, (LMPS'09), Irkutsk, Russia, 2009.

[3] C., Engels, et.al., "Integrated Optimization of Distribution System Planning and Transition into New Grid Structures", CIRED Workshop 2012, Lisboa, 2012.

[4] Wollenberg, Bruce; WOOD, Allen: Power Generation, Operation and Control. Second Edition, New-York: Wiley, 1996

[5] Kallweit, T.; Schwarz, U.; Spitzer, H.: „Asset Strategy Planning - Simulation als Basis fundierter und nachhaltiger Asset Strategien“, in: „et“, 55. Jg. (2005), Heft 7, S. 488-489;

[6] Gaul, A. J.; Spitzer, H.: „Asset Simulation - an approach to predict the long term monetary consequences of maintenance and renewal strategies for electrical grids", CIRED, 19th International Conference on Electricity Distribution, Wien, 21.24.5.2007, Paper 0668.

[7] Dutz, A., Spitzer, H., Schulterschluss zwischen Techniker und Kaufmann, et ENERGIEWIRTSCHAFTLICHE TAGESFRAGEN 59. Jg. (2009) Heft 4

[8] Spitzer, H., Engels, C., Dynamic Asset Simulation Risk Management am Beispiel der Energieversorgung, Riskconf, München, 2009.

[9] Forrester, J.W. 1961, Industrial Dynamics, OR: Productivity Press.

[10] Sterman, J.D.,2000, Business Dynamics, Systems Thinking and Modeling for a Complex World, Boston, MAet al.; Irwin/McGraw-Hill.

[12] Bäck, T.: Evolutionary Algorithms in Theory and Practice. New York: Oxford University Press, 1996.

(*) This work has been supported in the R\&D project "IO.Netz" funded by BMWi, grant no.: 03ET1071D 